\title{
Interactions between microbiota, diet/nutrients and immune/ inflammatory response in rheumatic diseases: focus on rheumatoid arthritis
}

\author{
Sabrina Paolino*, Greta Pacini", Massimo Patanè, Elisa Alessandri, Francesco Cattelan, \\ Federica Goegan, Carmen Pizzorni, Emanuele Gotelli, Maurizio Cutolo \\ Research Laboratory and Academic Unit of Clinical Rheumatology, Department of Internal Medicine, University of Genova, \\ IRCCS San Martino Polyclinic Hospital, Genova, Italy \\ * These authors contributed equally to this work.
}

\begin{abstract}
Rheumatic and musculoskeletal diseases (RMDs) are chronic systemic immune/inflammatory conditions characterized by the interaction between gene predisposition, autoimmunity and environmental factors. A growing scientific interest has focused on the role of diet in RMDs, suggesting its significant contribution to the pathogenesis and prognosis of these diseases. It is now clear that diet can directly modulate the immune response by providing a wide range of nutrients, which interfere with multiple pathways at both the gastro-intestinal and systemic level. Moreover, diet critically shapes the human gut microbiota, which is recognized to have a central role in the modulation of the immune response and in RMD pathogenesis. We hereby provide an in-depth analysis on the role of the microbiota in RMDs and on nutritional intervention as an integral part of a multidisciplinary approach. Particular attention will be given to the Mediterranean diet, as the only diet proven to support substantial benefits in RMD management.
\end{abstract}

Key words: connective tissue diseases, nutrition, microbiome, Mediterranean diet.

\section{Introduction}

Rheumatic and musculoskeletal diseases (RMDs) are chronic conditions characterized by a complex interaction between genetic, neuroendocrine and immunological assets with environmental factors (epigenetic modifiers).

Emerging data support a "dietary basis" for the inflammatory phenotype in RMDs, suggesting an integrated multidisciplinary approach to these conditions [1, 2].

A large number of studies confirm that diet could directly affect the immune response, stimulating or inhibiting inflammatory processes. Indeed, food-derived molecules interact with the gastrointestinal epithelial barrier, mucosal immune system and intestinal microbial flora, being responsible for both local and systemic modifications [1].

\section{Microbiota}

\section{The role of the microbiota in the immune response}

The human body is colonized by a multitude of microorganisms, which establish variable relationships with the host, behaving as symbionts, commensals or pathogens. This complex set of microorganisms, collectively called the microbiota, is mostly represented in the gastro-enteric tract [3]. The intestinal microbiota has coevolved with the host, establishing a mutual exchange relationship with it and providing a wide range of functions [4].

The broad spectrum of activities performed by the intestinal microbiota is centered on carbohydrate metabolism through fermentation processes and specif- 
ically on the degradation of indigestible dietary fibers, which are the main source of microbiota-accessible carbohydrates. An alternative energy source comes from the degradation of the mucus on the intestinal epithelial barrier, whose alterations become of crucial importance in the pathogenesis of many mucosal inflammatory diseases $[3,4]$.

The main end products of intestinal microbial fermentation are short-chain fatty acids (SCFAs), acetate, propionate and butyrate being the most extensively described both in the intestinal tract and in peripheral blood. This wide group of molecules is involved in several physiological processes, affecting colonocytes' morphology and functions, promoting immunity of the intestinal mucosa and protecting against colonization by pathogenic microbes [3, 4].

SCFAs derived from intestinal fermentation interact with multiple mechanisms at both local and systemic levels. Some molecules, such as butyrate, mainly exert a local action, nourishing the epithelial cells, regulating gut permeability and controlling mucosal inflammation. Other SCFAs have rather a systemic action, regulating inflammatory and neuroendocrine processes through complex signaling mechanisms [4].

Recent findings have even proposed that microbiota and, indirectly, dietary components may influence the availability of metabolites, their ability to cross the intestinal barrier and enter the circulation, producing long distance effects [5].

Within the broad spectrum of functions assigned to the microbiota, one of the most intriguing is undoubtedly its interaction with the immune system.

First of all, the microbiota plays a fundamental role in the differentiation of immune cells, more than $70 \%$ of them residing in the gastro-intestinal tract $[2,6]$. Additionally, alteration in the gut microbiota and modulation of local and systemic SCFAs modulate the innate and adaptive immune system by controlling $T$ cell differentiation, pro-inflammatory cytokine production, and dendritic cell and macrophage hematopoiesis and functionality $[4,7]$

\section{Microbiota modification and inflammatory diseases}

A growing body of literature has identified disruption of gut microbiota composition as a key factor in the pathogenesis of several diseases, first and foremost chronic inflammatory disorders [8-10]. It is currently recognized that any alteration in gut microbial composition, due to dietary factors or antibiotic therapies, creates a "dysbiotic state" affecting the abovementioned immune regulatory function and promoting a pro-inflammatory phenotype. This eventually leads to in- creased susceptibility to autoimmune diseases such as inflammatory bowel disease (IBD), systemic inflammatory arthritis and connective tissue diseases, as well as dysmetabolic conditions or even tumors [10].

In rheumatoid arthritis (RA), several studies in animal models and patients revealed that dysbiosis of the gut microbiota induces inflammatory autoimmune diseases and it is associated with disease progression [11]. Interestingly, RA patients fail to maintain the correct balance between "beneficial" and potentially "harmful" bacteria, leading to gastrointestinal reduction of Bifidobacteria and enhancement of the opportunistic species (Enterobacteria and Staphylococcus). A critical role has been accredited to increased concentration of Prevotella copri in the gut microhabitat, which seems to correlate with RA disease onset and severity. Thus, reduced Prevotella copri concentrations in RA patients have been shown to improve disease activity, paving the way for a new integrated approach to RA disease [11].

In spondyloarthritis (SpA), a great scientific interest has focused on the pathogenic role of altered gut permeability and skin and gut microbiota dysbiosis. Recent experimental models reported that HLA-B27 positive rats developed SpA-like disease only when exposed to particular pathogen-free enteric bacteria and not under germ-free conditions [12]. In psoriatic arthritis (PsA), gut dysbiosis has been shown to drive naïve CD4+ T cell differentiation into the Th-17 subset, promoting autoimmune arthritis in mouse models [13]. Moreover, an association between microbiota composition, toll-like receptors (TLRs) genetic polymorphisms and PsA susceptibility has been proposed [13].

In systemic lupus erythematosus (SLE), dysbiosis of the gut microbiota has extensively been reported for both mice and humans models. Notably, recent findings showed that the gut microbiota in SLE patients has a modified Firmicutes/Bacteroidetes ratio compared with that of healthy controls, with a reduced variety of species and a greater representation of Gram-negative bacteria $[14,15]$ although the underlying causes remain elusive. Recently, it has been suggested that factors other than inflammation or clinical features may be involved. The gut microbiota is known to influence the host metabolism, the production of short-chain fatty acids (SCFA).

\section{Probiotics supplementation}

The abundance and diversity of the microbiota as well as its relationship with the host are finely regulated by the interaction with various environmental factors. It is now recognized that not only genetic factors but also acquired conditions such as social and health status, dietary habits, exposure to pathogens and drugs can dynamically shape the composition of the microbiota [2, 4]. 
A prime subject of interest has certainly been probiotics use. According to the 2001 World Health Organization (WHO) definition, probiotics are living micro-organisms that, if administered in adequate amounts, confer a health benefit on the host [16].

Experimental studies of probiotics use in RMDs suggest that Lactobacilli are able to adhere to bowel epithelial cells, improving local immune function and thwarting pathogenic microorganisms invasion. In inflammatory arthritis, a modification in microbiota composition may act as a therapeutic target to improve the disease course [16]. In RA, Lactobacillus helveticus HY7801 administration has been demonstrated to stimulate anti-inflammatory cytokines such as IL-10, thus reducing the inflammatory immune response, and disease progression and severity [17].

\section{Diet}

\section{Impact of dietary changes on gut microbiota}

A growing number of studies have identified how diet plays a pivotal role in interaction with the microbiota, critically influencing its composition and function, with important implications in human health and diseases [18]. Dietary habits have drastically changed over the last few centuries, gradually reshaping a new dietary style. Notably, the dramatic reduction of dietary fiber intake has defined the so-called "fiber gap", or the inability to provide a sufficient nutrient supply for the intestinal microbiota, entailing the loss of its diversity and functions, with crucial pathophysiological implications $[18,19]$.

Since dietary habits are strongly impacted by the socio-economic context and lifestyle, the microbiota composition itself is affected, following a sort of biogeographic distribution [20]. The fiber gap is therefore particularly evident in western countries, where the diet is rich in simple sugars, fats and animal proteins, rather than in developing countries and rural areas that maintain carbohydrates and fiber-rich diets [20].

In this regard, an interesting study by De Filippo et al. has compared fecal microbiota of European children and rural African children, showing higher gut microbial diversity among African children and suggesting diet as a dominant variable over several ethno-geographic factors [21].

\section{Westernization of diet}

It has been widely demonstrated that the progressive "westernization" of the diet has increased the incidence and prevalence of several allergic and auto- immune diseases, as a result of a dysregulation of the immune system [20].

Modern western diets are characterized by high consumption of refined carbohydrates, added sugars, red and processed meats and salty foods. This kind of dietary habit can progressively induce a pro-inflammatory state promoting onset of autoimmune diseases [2, 20]. In addition, visceral adiposity influences the inflammatory status because the adipose tissue drives naïve myeloid cells into M1 pro-inflammatory macrophages [20].

Previous studies have suggested a potential link between salt consumption and the increased incidence of autoimmune diseases, particularly RA. Of note, a high concentration of sodium chloride induces an activation cascade of different signaling pathways converging in Th17 cell differentiation, overproduction of inflammatory cytokines (e.g. IFN- $\gamma$ and IL-17) and macrophage differentiation to M1 pro-inflammatory phenotype [22, 23].

\section{Diet as integrated therapy}

Given these considerations, it becomes evident that there is a crucial role of nutritional intervention in RMDs, which should be focused on the anti-inflammatory and antioxidant profile of nutrients $[2,18]$.

A large number of studies have confirmed that diet could modify the clinical history of RMDs by several mechanisms and dietary modification is of central concern for both patients and clinicians. Rheumatic patients often ask for nutritional counseling and sometimes engage in unorthodox diets with the hope of alleviating their physical complaints and improving the disease course. However, the results are often disappointing, thus necessitating expertise on dietary conduct as a complement to modern pharmacological treatment [2].

Despite the intense scientific production in the field, evidence is often discordant due to the lack of clinical trials and technical difficulties in their design because of randomization difficulties and compliance issues [1, 2]. Most of the scientific research has focused on RA, as the prototypical chronic inflammatory disease, in order to explore potential implications of dietary habits, and several diets have been considered for RMD management [24].

A growing number of studies concur that diets based on fruits, vegetables and whole grains prevent the development of several chronic diseases, while a diet rich in red and processed meats, refined grains and added sugars seems to increase the risk. Not surprisingly, the common dietary programs proposed to rheumatic patients include lacto-ovo-vegetarian or vegan diets, the Mediterranean diet (MedDiet), elemental eating plans and elimination diets $[2,24]$.

The effects of a vegan diet and elimination diet are still uncertain because of the limited availability of data 
and the higher rate of abandonment, probably linked to daily life practical constraints and reduced compliance with these strict regimens. Moreover, elimination diets result in the exclusion of one or more food components, thus leading to nutritional deficiencies, which are detrimental in chronic inflammatory disease $[2,24]$.

\section{Mediterranean diet}

Originating in the rural areas of countries of the upper Mediterranean region, the MedDiet is characterized by a great variety of foods. The MedDiet has been reported as the best-balanced and complete diet, providing a wide range of macro- and micronutrients. Additionally, the MedDiet is pleasant, ensuring excellent patient compliance [2]. The MedDiet fully satisfies the required nutrient supply (Table I), providing a great nutritional balance, characterized by consistent intake of extra-virgin olive oil, vegetables, fruits, nuts and legumes, whole grains, and fish and by moderate consumption of eggs, dairy products, lean meats, and alcohol. At the same time, the MedDiet contains small amounts of saturated fats, red meat, refined carbohydrates, and sweets. Globally, the MedDiet supplies a balanced mix of nutrients that provides antioxidant, anti-inflammatory and prebiotic effects [2].

The MedDiet is the only proposed diet for RMDs to have been confirmed by a wide range of clinical and observational studies. Nurses' Health Study I and II, which are among the largest prospective investigations into the risk factors for major chronic diseases in women, confirmed its beneficial effects in reducing pain and increasing physical function in RA patients [24]. In a pilot trial conducted by McKellar et al., RA females following a MedDiet had a better disease outcome during their follow-up, such as improvement in pain and physical function at 3 months, and reduction in global VAS and morning stiffness at 6 months [25].

\section{Nutrients}

\section{Antioxidant profile of nutrients}

One of the milestones of the MedDiet is the high amount of antioxidant substances. As known, dispropor-

Table I. Dietary reference values for nutrients according to European Food Safety Authority 2017 in healthy conditions (modified with permission of Cutolo)

\begin{tabular}{|c|c|c|c|c|c|c|c|}
\hline \multirow[t]{4}{*}{ Nutrients } & \multicolumn{7}{|c|}{ Gender and physiological state } \\
\hline & \multirow{3}{*}{$\begin{array}{l}\text { >18-year } \\
\text { old male }\end{array}$} & \multicolumn{6}{|c|}{ > 18-year old female } \\
\hline & & \multicolumn{4}{|c|}{ Pregnancy } & \multicolumn{2}{|c|}{ Lactation } \\
\hline & & $\begin{array}{c}\text { Not } \\
\text { pregnant }\end{array}$ & $\begin{array}{c}1^{\text {st }} \\
\text { trimester }\end{array}$ & $\begin{array}{c}2^{\text {nd }} \\
\text { trimester }\end{array}$ & $\begin{array}{c}3^{\text {rd }} \\
\text { trimester }\end{array}$ & $\leq 6 \mathrm{mo} \mathrm{PP}$ & $>6 \mathrm{mo} \mathrm{PP}$ \\
\hline \multicolumn{8}{|l|}{ Carbohydrates } \\
\hline Total $^{a}(\mathrm{E} \%)$ & $45-60$ & $45-60$ & \multicolumn{3}{|c|}{$45-60$} & \multicolumn{2}{|c|}{$45-60$} \\
\hline Dietary fibers ${ }^{b}$ (g/day) & 25 & 25 & \multicolumn{3}{|c|}{25} & \multicolumn{2}{|c|}{25} \\
\hline \multicolumn{8}{|l|}{ Lipids } \\
\hline Total $^{\mathrm{a}}(\mathrm{E} \%)$ & $20-35$ & $20-35$ & \multicolumn{3}{|c|}{$20-35$} & \multicolumn{2}{|c|}{$20-35$} \\
\hline $\mathrm{SFA}^{\mathrm{b}}$ (mg/day) & ALAP & ALAP & \multicolumn{3}{|c|}{ ALAP } & \multicolumn{2}{|c|}{ ALAP } \\
\hline $\mathrm{LA}^{\mathrm{b}}(\mathrm{E} \%)$ & 4 & 4 & \multicolumn{3}{|c|}{4} & \multicolumn{2}{|c|}{4} \\
\hline $\mathrm{ALA}^{\mathrm{b}}(\mathrm{E} \%)$ & 0.5 & 0.5 & \multicolumn{3}{|c|}{0.5} & \multicolumn{2}{|c|}{0.5} \\
\hline$E P A+\mathrm{DHA}^{\mathrm{b}}(\mathrm{mg} /$ day $)$ & 250 & 250 & \multicolumn{3}{|c|}{250} & \multicolumn{2}{|c|}{250} \\
\hline $\mathrm{DHA}^{\mathrm{b}}$ (mg/day) & & & \multicolumn{3}{|c|}{$+100-200^{c}$} & \multicolumn{2}{|c|}{$+100-200^{c}$} \\
\hline TFA $^{b}$ (mg/day) & ALAP & ALAP & \multicolumn{3}{|c|}{ ALAP } & \multicolumn{2}{|c|}{ ALAP } \\
\hline \multicolumn{8}{|l|}{ Proteins } \\
\hline $\mathrm{AR}^{\mathrm{d}}$ (g/kg/day) & 0.66 & 0.66 & $+0.52 \mathrm{~g} /$ daye $^{\mathrm{e}}$ & $+7.2 \mathrm{~g} /$ day $^{\mathrm{e}}$ & +23 g/daye & +10 g/day & +15 g/day \\
\hline $\mathrm{PRI}^{\mathrm{d}}$ (g/kg/day) & 0.83 & 0.83 & $+1 \mathrm{~g} /$ day $^{\mathrm{f}}$ & $+9 \mathrm{~g} /$ day $^{f}$ & +28 g/day ${ }^{f}$ & +19 g/day ${ }^{f}$ & +23 g/day ${ }^{f}$ \\
\hline Waterb,g (I/day) & 2.5 & 2.0 & & 2.3 & & & \\
\hline
\end{tabular}

$A L A$ - $\alpha$-linolenic acid, ALAP - as low as possible, AR - average requirement, DHA - docosahexaenoic acid, E\% - percentage of energy intake, EPA - eicosapentaenoic acid, LA - linoleic acid, PP - postpartum, PRI-population reference intake, SFA - saturated fatty acids, TFA - trans-fatty acids, ${ }^{a} R I$ - reference intake range, ${ }^{b} \mathrm{Al}$ - adequate intake, ${ }^{c}$ in addition to combined intakes of EPA and DHA of $250 \mathrm{mg} /$ day, ${ }^{d}$ to be multiplied by reference body weights to calculate values in $\mathrm{g} /$ day, ${ }^{~}$ in addition to AR for protein of non-pregnant, non-lactating women, ${ }^{f}$ in addition to PRI for protein of non-pregnant, non-lactating women, $g$ includes water from beverages of all kinds, including drinking and mineral water, and from food moisture. 
tionate production of free radicals in the cells increases oxidative stress, being responsible for many chronic conditions such as cancer, cardiovascular complications, autoimmune diseases and ageing [2]. In order to neutralize oxidative stress, cells could rely on endogenous defense mechanisms as well as exogenous substances such as phytochemicals and vitamins. Dietary antioxidants, mainly contained in fruits, vegetables, nuts, cocoa and cereals and usually considered to have little nutritional value, are actually a heterogeneous group of bioactive compounds with a strong anti-inflammatory function and a good antioxidant profile [26].

For instance, red fruits, such as blueberries, raspberries and strawberries, are rich in bioactive compounds with antioxidant anti-inflammatory and analgesic effects. Interestingly, these nutrients have been recently reported to reduce pain and inflammation in an experimental model and in human clinical studies of arthritis [26].

Grapes, cherries and red wine contain a high concentration of resveratrol, a specific antioxidant rapidly absorbed in the gastrointestinal tract that inhibits the activity of the nuclear factor $\mathrm{\kappa B}(\mathrm{NF}-\mathrm{kB})$, exerting a protective inflammation [27].

In RA in vitro models, resveratrol seemed to ameliorate synovitis by acting on inflammatory cell infiltration and angiogenesis, reducing oxidative stress and inducing cell apoptosis in synovial tissue. In inflammatory animal models resveratrol suppressed Th-17 cell expansion and deriving pro-inflammatory cytokine production, decreased serum levels of rheumatoid factor, matrix metalloproteinase 3 (MMP-3) and increased anti-inflammatory mediators such as IL-10 [27].

\section{Anti-inflammatory profile of nutrients}

Spices, which have always been important components in the MedDiet, contain several substances, such as curcumin and capsaicin, which exert a regulatory effect on oxidative stress and inflammation.

Turmeric root, which gives Indian curry its characteristic yellow color, has been used for thousands of years in Ayurvedic and Chinese medicine to treat a variety of clinical conditions, but has only recently received scientific attention for its powerful antioxidant and anti-inflammatory properties [28].

Curcumin suppresses the inflammatory cascade by blocking the NF-kB pathway and other pro-inflammatory pathways including cyclooxygenase (COX) 2. In vitro, curcumin downregulates the immune response by blocking IL-1 and IL-6 expression in RA synoviocytes [1]. Beneficial properties of curcuma have even been supported by previously reported RCTs conducted in RA where a curcumin-rich diet resulted in an overall disease activity improvement comparable to that observed with NSAIDs [1].

Other interesting findings have emerged on the role of the epigallocatechin 3-gallate (EGCG), the most abundant catechin in tea, belonging to the family of polyphenols, which seems to interfere with the NF-kB pathway, having potential implications for physiologic and pathologic conditions [29].

Studies conducted on RA showed that EGCG downregulates anti-apoptotic proteins such as $\mathrm{Mcl}-1$ in synovial fibroblasts, increases apoptosis and reduces MMP-3 production, bringing positive effects on bone and cartilage [29].

\section{Appropriate lipid intake}

Assuming the aforementioned pro-inflammatory action of visceral adiposity, a proper anti-inflammatory diet should also include a good fat profile with a low intake of saturated fats and an optimal ratio of n-6 to n-3 polyunsaturated fatty acids (PUFAs) [30].

Omega-3 PUFAs have been shown to modulate immune system activity through immunomodulatory and anti-inflammatory properties. In particular, they inhibit oxygen free radical production and several redox-sensitive transcription factors, including NF-kB, which is involved in various pro-inflammatory pathways [30].

Diet-derived PUFAs are able to interact with a multitude of inflammatory processes such as leukocyte chemotaxis and production of prostaglandins and leukotrienes, which are major mediators of inflammation [30]. As is known, fish is one of the foods richest in long-chain n-3 PUFAs. Interestingly, an inverse correlation between fish consumption and RA incidence has been reported.

Specifically, long-term fish consumption (>0.21 g/day, corresponding to at least one portion per week of fatty fish, e.g. salmon, or four portions per week of lean fish, e.g. cod) was associated with a 52\% decreased risk of developing RA in a recent prospective cohort study [31].

\section{The role of extra virgin olive oil}

A crucial component of MedDiet is certainly extra virgin olive oil (EVOO). EVOO composition mainly consists in a saponifiable fraction made up of fatty acids, notably monounsaturated fatty acids (MUFAs) such as oleic acid, PUFAs such as linoleic acid, and saturated fatty acids (SFAs) such as palmitic acid. Traditionally, the beneficial effects of EVOO on the immune system have been related to the high content of MUFAs [2].

In mouse models, food supplementation with EVOO has been associated with reduced expression of $M 1$ pro-inflammatory macrophages and increased expression of M2 anti-inflammatory macrophages in bone 
marrow. In addition, EVOO seems to prevent Janus kinase/signal transducer activation as well as NF-kB signaling pathways [32].

In an RA murine model, EVOO-enriched diets have been shown to improve disease activity, reducing joint edema and cartilage destruction. In fact, mice receiving higher doses of EVOO had significantly lower levels of pro-inflammatory cytokines (TNF- $\alpha, \mathrm{IL}-1 \beta$ and IL-17) and MMP-3 compared with controls [32].

Additionally, a large recent study has shown that a high intake of MUFAs can be a predictive factor of remission in RA, assessed by the disease activity score in 28 joints with the erythrocyte sedimentation rate (DAS28-ERS) [33].

Further investigations identified oleocanthal as the main factor responsible for the nutritional properties of EVOO. This natural phenolic compound has been found to have potent anti-inflammatory and antioxidant properties, mainly derived from cyclooxygenase COX-1 and 2 inhibition, assuming a potential therapeutic interest [34].

\section{The "myoprotective effect" and the effect on bone metabolism}

Along with anti-inflammatory, anti-oxidant and general benefits on metabolic and cardiovascular function, the MedDiet has recently been recognized as exerting a so-called "myoprotective effect". Much of the current literature confirms the positive role of the MedDiet on muscle aging and sarcopenia. This would be ensured not just by protein intake - relatively low in comparison to other dietary patterns - but by the potent anti-inflammatory, anti-oxidant and pro-anabolic function of nutrients [35]. Additionally, a recent meta-analysis has reported that high adherence to the MedDiet positively interacts with bone metabolism, being associated with a $21 \%$ reduced risk of hip fracture as well with a higher bone mineral density at the lumbar spine, hip and whole body [36]. These findings may provide useful implications in the multidisciplinary approach to RMDs, especially in the case of elderly and frail patients.

\section{Conclusions}

RMDs are chronic systemic immune/inflammatory conditions characterized by an intricate multifactorial framework of genetic predisposition and environmental factors, including the effects of chrononutrition, which is linked to the circadian rhythms [37-39].

In addition, it has been widely demonstrated and it is increasingly evident that dietary habits play an important role in the course and prognosis of these diseases.
The Mediterranean diet is the only one that has proven to support a substantial health benefit among the different dietary approaches proposed for RMDs [2].

Unfortunately, the impact of nutrition on chronic musculoskeletal diseases remains poorly addressed in clinical practice. Still, proper nutritional counseling can be a valuable weapon to greatly improve disease outcomes, since it is inexpensive and easily integrated in any social and economic background.

It is therefore the task of the good rheumatologist to be concerned with this fast-growing field and to educate on the central value of nutrition in the therapeutic approach [2].

The authors declare no conflicts of interest.

\section{References}

1. Dahan S, Segal Y, Shoenfeld Y. Dietary factors in rheumatic autoimmune diseases: A recipe for therapy? Nat Rev Rheumatol 2017; 13: 348-358.

2. Cutolo M, Nikiphorou E. Don't neglect nutrition in rheumatoid arthritis! RMD Open 2018; 4: e000591.

3. Han M, Wang C, Liu P, et al. Dietary Fiber Gap and Host Gut Microbiota. Protein Pept Lett 2017; 24: 388-396.

4. Abdollahi-Roodsaz S, Abramson SB, Scher JU. The metabolic role of the gut microbiota in health and rheumatic disease: mechanisms and interventions. Nat Rev Rheumatol 2016; 12: 446-455.

5. Schroeder BO, Bäckhed F. Signals from the gut microbiota to distant organs in physiology and disease. Nat Med 2016; 22: 1079-1089.

6. Zuo T, Kamm MA, Colombel JF, et al. Urbanization and the gut microbiota in health and inflammatory bowel disease. Nat Rev Gastroenterol Hepatol 2018; 15: 440-452.

7. Trompette A, Gollwitzer ES, Yadava K, et al. Gut microbiota metabolism of dietary fiber influences allergic airway disease and hematopoiesis. Nat Med 2014; 20: 159-166.

8. Van de Wiele T, Van Praet JT, Marzorati M, et al. How the Microbiota Shapes Rheumatic Diseases. Nat Rev Rheumatol 2016; 12: 398-411.

9. Zechner EL. Inflammatory disease caused by intestinal pathobionts. Curr Opin Microbiol 2017; 35: 64-69.

10. Lynch SV, Pedersen O. The Human Intestinal Microbiome in Health and Disease. N Engl J Med 2016; 375: 2369-2379.

11. Alpizar-Rodriguez D, Lesker TR, Gronow A, et al. Prevotella copri in individuals at risk for rheumatoid arthritis. Ann Rheum Dis 2019; 78: 590-593.

12. Jethwa $\mathrm{H}$, Abraham $\mathrm{S}$. The evidence for microbiome manipulation in inflammatory arthritis. Rheumatology (Oxford) 2017; 56: 1452-1462.

13. Chimenti MS, Perricone C, Novelli L, et al. Interaction between microbiome and host genetics in psoriatic arthritis. Autoimmun Rev 2018; 17: 276-283.

14. Rodríguez-Carrio J, López P, Sánchez B, et al. Intestinal Dysbiosis Is Associated with Altered Short-Chain Fatty Acids and Se- 
rum-Free Fatty Acids in Systemic Lupus Erythematosus. Front Immunol 2017; 8: 23.

15. Luo XM, Edwards MR, Mu Q, et al. Gut Microbiota in Human Systemic Lupus Erythematosus and a Mouse Model of Lupus. Appl Environ Microbiol 2017; 84: e02288-17.

16. Liu Y, Alookaran JJ, Rhoads JM. Probiotics in Autoimmune and Inflammatory Disorders. Nutrients 2018; 10: e1537.

17. Kim JE, Chang SC, Kim GC, et al. Lactobacillus helveticus suppresses experimental rheumatoid arthritis by reducing inflammatory T cell responses. J Funct Foods 2015; 13: 350-362.

18. Derrien M, Veiga P. Rethinking Diet to Aid Human-Microbe Symbiosis. Trends Microbiol 2017; 25: 100-112.

19. Deehan EC, Walter J. The Fiber Gap and the Disappearing Gut Microbiome: Implications for Human Nutrition. Trends Endocrinol Metab 2016; 27: 239-242.

20.Zinöcker M, Lindseth I. The Western Diet - Microbiome-Host Interaction and Its Role in Metabolic Disease. Nutrients 2018 10: e365.

21. De Filippo C, Cavalieri D, Di Paola M, et al. Impact of diet in shaping gut microbiota revealed by a comparative study in children from Europe and rural Africa. Proc Natl Acad Sci USA 2010; 107: 14691-14696.

22. Wilck MG, Matus SM, Kearney SM, et al. Salt-responsive gut commensal modulates TH17 axis and disease. Nature 2017 551: 585-589.

23. Sharif K, Amital H, Shoenfeld Y. The role of dietary sodium in autoimmune diseases: The salty truth. Autoimmun Rev 2018, 17: 1069-1073.

24. Hu Y, Sparks JA, Malspeis S, et al. Long-term dietary quality and risk of developing rheumatoid arthritis in women. Ann Rheum Dis 2017; 76: 1357-1364.

25. McKellar G, Morrison E, McEntegart A. A pilot study of a Mediterranean-type diet intervention in female patients with rheumatoid arthritis living in areas of social deprivation in Glasgow. Ann Rheum Dis 2007; 66: 1239-1243.

26. Khanna S, Jaiswal KS, Gupta B. Managing Rheumatoid Arthritis with Dietary Interventions. Front Nutr 2017; 4: 52.

27. Oliveira A, Monteiro V, Navegantes-Lima K, et al. Resveratrol Role in Autoimmune Disease - A Mini-Review. Nutrients 2017; 9: e1306.
28. Kunnumakkara AB, Bordoloi D, Padmavathi G, et al. Curcumin, the golden nutraceutical: multitargeting for multiple chronic diseases. Br J Pharmacol 2017; 174: 1325-1348.

29. Kim H-S, Quon MJ, Kim J. New insights into the mechanisms of polyphenols beyond antioxidant properties; lessons from the green tea polyphenol, epigallocatechin 3-gallate. Redox Biol 2014; 2: 187-195.

30. Gioxari A, Kaliora AC, Marantidou F, et al. Intake of $\omega$-3 polyunsaturated fatty acids in patients with rheumatoid arthritis: A systematic review and meta-analysis. Nutrition 2018; 45: 114-124.e4.

31. Di Giuseppe D, Crippa A, Orsini N, et al. Fish consumption and risk of rheumatoid arthritis: a dose-response meta-analysis. Arthritis Res Ther 2014; 16: 446.

32. Rosillo MA, Sánchez-Hidalgo M, Sánchez-Fidalgo S, et al. Dietary extra-virgin olive oil prevents inflammatory response and cartilage matrix degradation in murine collagen-induced arthritis. Eur J Nutr 2016; 55: 315-325.

33. Matsumoto Y, Sugioka Y, Tada M, et al. Monounsaturated fatty acids might be key factors in the Mediterranean diet that suppress rheumatoid arthritis disease activity: The TOMORROW study. Clin Nutr 2018; 37: 675-680.

34. Pang K-L, Chin K-Y. The Biological Activities of Oleocanthal from a Molecular Perspective. Nutrients 2018; 10: e570.

35. Granic A, Sayer AA, Robinson SM. Dietary Patterns, Skeletal Muscle Health, and Sarcopenia in Older Adults. Nutrients 2019; 11: e745.

36. Malmir H, Saneei P, Larijani B, et al. Adherence to Mediterranean diet in relation to bone mineral density and risk of fracture: a systematic review and meta-analysis of observational studies. Eur J Nutr 2018; 57: 2147-2160.

37. Cutolo M, Straub RH. Circadian rhythms in arthritis: Hormonal effects on the immune/inflammatory reaction. Autoimmunity Rev 2008; 7: 223-228.

38. Sulli A, Maestroni GJ, Villaggio B, et al. Melatonin serum levels in rheumatoid arthritis. Ann NY Acad Sci 2002; 966: 276-283.

39. Masi AT, Bijlsma JW, Chikanza IC, et al. Neuroendocrine, immunologic, and microvascular systems interactions in rheumatoid arthritis: physiopathogenetic and therapeutic perspectives. Semin Arthritis Rheum 1999; 29: 65-68. 